\title{
Adenylyl Cyclase 1 Is Required for Ethanol-Induced Locomotor Sensitization and Associated Increases in NMDA Receptor Phosphorylation and Function in the Dorsal Medial Striatum
}

\author{
Kelly E. Bosse, Max F. Oginsky, Laura L. Susick, Sailesh Ramalingam, Carrie R. Ferrario, \\ and Alana C. Conti
}

Research \& Development, John D. Dingell VA Medical Center, Detroit, Michigan (K.E.B., L.L.S., S.R., A.C.C.); Department of Neurosurgery, Wayne State University School of Medicine, Detroit, Michigan (K.E.B., L.L.S., S.R., A.C.C.); and Department of Pharmacology, University of Michigan Medical School, Ann Arbor, Michigan (M.F.O., C.R.F.)

Received April 25, 2017; accepted August 8, 2017

\begin{abstract}
Neuroadaptive responses to chronic ethanol, such as behavioral sensitization, are associated with $N$-methyl-D-aspartate receptor (NMDAR) recruitment. Ethanol enhances GluN2B-containing NMDAR function and phosphorylation (Tyr-1472) of the GluN2BNMDAR subunit in the dorsal medial striatum (DMS) through a

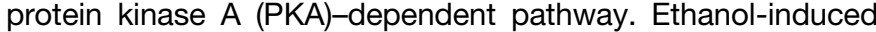
phosphorylation of PKA substrates is partially mediated by calcium-stimulated adenylyl cyclase 1 (AC1), which is enriched in the dorsal striatum. As such, AC1 is poised as an upstream modulator of ethanol-induced DMS neuroadaptations that promote drug responding, and thus represents a therapeutic target. Our hypothesis is that loss of AC1 activity will prevent ethanolinduced locomotor sensitization and associated DMS GluN2BNMDAR adaptations. We evaluated AC1's contribution to ethanol-evoked locomotor responses and DMS GluN2B-NMDAR phosphorylation and function using $A C 1$ knockout (AC1KO) mice.
\end{abstract}

Results were mechanistically validated with the AC1 inhibitor, NB001. Acute ethanol $(2.0 \mathrm{~g} / \mathrm{kg})$ locomotor responses in AC1KO and wild-type (WT) mice pretreated with NB001 $(10 \mathrm{mg} / \mathrm{kg})$ were comparable to WT ethanol controls. However, repeated ethanol treatment (10 days, $2.5 \mathrm{~g} / \mathrm{kg}$ ) failed to produce sensitization in AC1KO or NB001 pretreated mice, as observed in WT ethanol controls, following challenge exposure $(2.0 \mathrm{~g} / \mathrm{kg})$. Repeated exposure to ethanol in the sensitization procedure significantly increased pTyr-1472 GluN2B levels and GluN2B-containing NMDAR transmission in the DMS of WT mice. Loss of AC1 signaling impaired ethanol-induced increases in DMS pGluN2B levels and NMDAR-mediated transmission. Together, these data support a critical and specific role for $\mathrm{AC} 1$ in striatal signaling that mediates ethanol-induced behavioral sensitization, and identify GluN2B-containing NMDARs as an important AC1 target.

\section{Introduction}

Ethanol-induced locomotor sensitization is a manifestation of long-lasting neuroadaptations in striatal reward networks, which overlap with neural substrates contributing to ethanol self-administration (Steketee and Kalivas, 2011). Clinical studies have similarly identified heightened reward sensitivity and accompanying striatal activation following ethanol exposure (Boileau et al., 2003; Grodin et al., 2016), supporting

This work was supported with resources at the John D. Dingell VA Medica Center and Department of Pharmacology, University of Michigan, and funds from the Wayne State University Department of Neurosurgery (A.C.C.) and National Institutes of Health National Institute on Drug Abuse [Grant T32DA007268, to M.F.O.]

Part of this work was presented as follows: Bosse KE, Susick LL, Ramalingam S, and Conti AC (2015) Role for adenylyl cyclase 1 in ethanolmediated locomotor sensitization and striatal NMDA glutamate receptor regulation. Research Society on Alcoholism National Meeting; 2015 Jun 20-24; San Antonio, TX

The authors declare no competing conflict of interest.

https://doi.org/10.1124/jpet.117.242321. the validity of sensitization as a preclinical model to recapitulate neural adaptations that promote drug responding (Lessov et al., 2001; Abrahao et al., 2013). Glutamatergic transmission through $N$-methyl-D-aspartate receptors (NMDARs) is particularly implicated in the cellular mechanisms governing both ethanol-evoked drug reinstatement and behavioral sensitization (Kotlinska et al., 2006; Steketee and Kalivas, 2011; Abrahao et al., 2013). Chronic ethanol exposure potentiates transmission mediated by postsynaptic GluN2B-containing NMDARs in the dorsal medial striatum (DMS) (Wang et al., 2010). This increase in NMDAR-mediated transmission is associated with enhanced phosphorylation of Tyr-1472 on the GluN2B subunit that is indirectly mediated by protein kinase A (PKA) (Wang et al., 2010; Darcq et al., 2014). While inhibition of GluN2B-NMDARs specifically in the DMS decreases ethanol self-administration (Wang et al., 2010), targeting NMDARs for treatment and prevention of alcoholism poses a challenge for designing safe, selective, and efficacious drugs due to the biologically ubiquitous nature of NMDAR-mediated transmission

ABBREVIATIONS: AC, adenylyl cyclase; AC1KO, adenylyl cyclase 1 knockout; ANOVA, analysis of variance; DLS, dorsal lateral striatum; DMS, dorsal medial striatum; eEPSC, evoked postsynaptic current; NAc, nucleus accumbens; NMDAR, $N$-methyl-D-aspartate receptor; PKA, protein kinase A; RM, repeated measures; WT, wild type. 
(Holmes et al., 2013; Spanagel and Vengeliene, 2013). Therefore, upstream modulators of GluN2B-NMDARs in the DMS, which are expressly associated with these ethanol neuroadaptations, have become more attractive targets and should be investigated.

Ethanol-mediated induction of cAMP-PKA signaling (Ron and Messing, 2013) occurs through a calcium-dependent process in the striatum (Baliño et al., 2014). The only neuronal adenylyl cyclase (AC) isoforms that catalyze cAMP upon calcium/calmodulin activation are AC1 and AC8 (Wang and Storm, 2003), which were selectively upregulated in the striatum of human alcoholic brains (Yamamoto et al., 2001), underscoring a specific role for these ACs in alcoholism. Animal models support calcium-stimulated ACs in ethanol action, since genetic deletion of AC1 and AC8 increased sedation, decreased voluntary consumption, and reduced locomotor stimulation to ethanol (Maas et al., 2005; Conti et al., 2012). Although ethanol does not directly stimulate AC1 or AC8 activity (Yoshimura and Tabakoff, 1995; Maas et al., 2005), the observed increase in ethanol-induced sedation in AC1/AC8-deficient mice was accompanied by impaired ethanol-induced phosphorylation of a subset of neuronal PKA targets, driven specifically by loss of AC1 (Conti et al., 2009). Since AC1 expression is enriched in the dorsal, compared with the ventral, striatum (Olsen et al., 2008 ), its striatal localization and role as a calcium detector poises AC1 to modulate the activity-dependent neuroplastic events in the DMS evoked by repeated ethanol, such as the PKA-dependent phosphorylation of GluN2B-NMDARs.

This study tests the hypothesis that loss of AC1 activity will prevent ethanol-induced locomotor sensitization and associated molecular adaptations in DMS GluN2B-NMDAR transmission and phosphorylation. Locomotor sensitization and changes in DMS GluN2B-NMDAR Tyr-1472 phosphorylation and NMDAR-mediated transmission were assessed in wildtype (WT) and $\mathrm{AC} 1$ knockout $(\mathrm{AC} 1 \mathrm{KO})$ mice after repeated saline or ethanol exposure. A selective pharmacological AC1 inhibitor NB001 was used to mechanistically validate the effect of AC1 in ethanol-induced locomotor sensitization and corresponding increases in GluN2B phosphorylation. Together, these findings support an imperative role for AC1 in the sensitizing effects of ethanol, and associated upregulation in NMDAR function localized in the DMS.

\section{Materials and Methods}

Animals. Age-matched progeny of AC1KO homozygous mutants and WT (C57BL/6) mice (male, 8-12 weeks old) were bred and raised in-house. Mice were maintained in standard microisolator cages under controlled conditions $\left(\sim 24^{\circ} \mathrm{C}\right.$; lights on $7 \mathrm{AM}$ to $7 \mathrm{PM}$; $35 \%-40 \%$ humidity) with constant free access to food and water. All efforts were made to minimize animal suffering, to reduce the number of animals used, and to research potential applicable alternatives to in vivo techniques. All procedures were approved by the Wayne State University and University of Michigan Institutional Animal Care and Use Committees. Animal care and use followed National Institutes of Health Animal Care guidelines (https://grants.nih.gov/grants/olaw/Guide-forthe-Care-and-Use-of-Laboratory-Animals.pdf) and was overseen by the Association for Assessment and Accreditation of Laboratory Animal Care-accredited facilities at Wayne State University and the University of Michigan.

Ethanol-Induced Locomotor Sensitization. Locomotor activity was recorded using an Opto-M3 Activity Meter (Columbus Instruments, Columbus, $\mathrm{OH}$ ). Sequential horizontal beam breaks (1-minute intervals) of parallel infrared emitter pairs were measured in novel static chambers containing standard microisolator cages. C57Bl/6 mice are known to display enhanced novelty responding (Cabib et al., 2002; Rose et al., 2013) and brief, transient elevations in activity to low-dose ethanol administration (Crabbe et al., 1982; Lessov et al., 2001; Hefner and Holmes, 2007; Melón and Boehm, 2011; Conti et al., 2012), which impedes the ability to detect excitatory locomotor responses to ethanol in this strain. To augment habituation to the testing environment and injection procedure, mice were brought to the room 1 hour prior to daily testing and exposed to at least three daily acclimation sessions preceding ethanol administration. During each acclimation session, mice received a single intraperitoneal sterile saline injection $(0.9 \% \mathrm{NaCl}$ solution, volume equivalent to that of a 2.0 $\mathrm{g} / \mathrm{kg}$ ethanol dose), which also served as a measurement of baseline activity. The general sensitization procedure and a 10 -minute recording session were used to capture the temporal pattern of ethanolinduced ambulatory changes in C57BL/6 mice based on the literature (Crabbe et al., 1982; Lessov et al., 2001; Hefner and Holmes, 2007; Melón and Boehm, 2011; Conti et al., 2012). Ethanol (200 proof; Fisher Scientific, Waltham, MA) and NB001 (SML0060; Sigma-Aldrich, St. Louis, MO) were diluted in sterile saline for injection to $20 \%(\mathrm{v} / \mathrm{v})$ and $1 \mathrm{mg} / \mathrm{ml}$ solutions, respectively. Following baseline assessment, mice were randomly assigned to a treatment group, which received either saline or $2.0 \mathrm{~g} / \mathrm{kg}$ ethanol (i.p.) to measure acute locomotor activity. Mice received home cage injections of saline or $2.5 \mathrm{~g} / \mathrm{kg}$ ethanol (i.p.), according to their initial randomized treatment group, for 10 consecutive days. Expression of locomotor sensitization was measured after a challenge dose of saline or $2.0 \mathrm{~g} / \mathrm{kg}$ ethanol (i.p.). In the cohort that received NB001, mice were injected with saline or NB001 (10 mg/kg, i.p.) 30 minutes prior to daily saline or ethanol injections throughout testing (including baseline assessment) to yield four treatment groups (saline + saline, saline + ethanol, NB001 + saline, and NB001 + ethanol). Ambulatory activity was reported as percentage change relative to respective saline-only controls.

Tissue Collection and Immunoblotting. Brains were removed either 20 minutes or 24 hours after the last saline or ethanol injection, flash frozen in liquid nitrogen, and stored at $-80^{\circ} \mathrm{C}$. Frozen brains were partly thawed on ice, sectioned into $2 \mathrm{~mm}$ coronal slices on an icecold brain matrix, and a 1-mm biopsy punch was used to isolate the DMS, dorsal lateral striatum (DLS), and nucleus accumbens (NAc) from the slice on a solid block of $\mathrm{CO}_{2}$. Dissections were guided by neuroanatomical landmarks (Paxinos and Franklin, 2001). Punches from one hemisphere were homogenized with a buffer composition of $2 \mathrm{M}$ thiourea, $7 \mathrm{M}$ urea, $30 \mathrm{mM}$ Tris ( $\mathrm{pH} 8.5$ ), 4\% (w/v) 3-[(3-cholamidopropyl)dimethylammonio]-1-propanesulfonate, and protease and phosphatase inhibitors); centrifuged $(8000 \mathrm{~g})$ for 10 minutes $\left(4^{\circ} \mathrm{C}\right)$; and the supernatant was stored at $-80^{\circ} \mathrm{C}$ until analyzed. Equal amounts of protein $(10 \mu \mathrm{g})$ were separated using SDS-PAGE $(4 \%-12 \%$ Bis-Tris NuPAGE gel [Invitrogen, Carlsbad, CA]), transferred to a nitrocellulose membrane, and probed with primary antibodies against pTyr-1472-GluN2B-NMDAR (1:2000, 4208; Cell Signaling Technology, Danvers, MA), GluN2B-NMDAR (1:2000, 4212; Cell Signaling Technology), and actin (1:5000, A5060; Sigma-Aldrich). Primary antibodies were detected using horseradish peroxidase-conjugated goat anti-rabbit secondary antibodies and signals were visualized using chemiluminescence. Densitometric analysis was performed using Image J Software. pTyr-1472-GluN2B-NMDAR signals were normalized to corresponding total GluN2B-NMDAR bands and total GluN2B-NMDAR signals were normalized to corresponding actin bands. Normalized signal intensity for each was averaged within groups.

Slice Preparation and Electrophysiology. Whole cell patchclamp recordings of DMS medium spiny neurons were made in slices obtained 20-26 hours following the last saline or ethanol injection. Mice were anesthetized with isoflurane, and brains were rapidly removed and placed in ice-cold oxygenated $\left(95 \% \mathrm{O}_{2}-5 \% \mathrm{CO}_{2}\right)$ artificial cerebrospinal fluid containing (in millimolars): $125 \mathrm{NaCl}, 25 \mathrm{NaHCO}_{3}$, 12.5 glucose, $1.25 \mathrm{NaH}_{2} \mathrm{PO}_{4}, 3.5 \mathrm{KCl}, 1 \mathrm{~L}$-ascorbic acid, $0.5 \mathrm{CaCl}_{2}$, $3 \mathrm{MgCl}_{2}, 305 \mathrm{mOsm}, \mathrm{pH}$ 7.4. Coronal sections containing DMS 
$(250 \mu \mathrm{m})$ were cut with a vibratome (Leica Biosystems, Wetzlar, Germany) and allowed to recover in oxygenated artificial cerebrospinal fluid $\left(37^{\circ} \mathrm{C}, 40\right.$ minutes) prior to recordings. For the recording artificial cerebrospinal fluid $(2 \mathrm{ml} / \mathrm{min}), \mathrm{CaCl}_{2}$ was increased to $2.5 \mathrm{mM}$ and $\mathrm{MgCl}_{2}$ was decreased to $1 \mathrm{mM}$. The GABA receptor antagonist picrotoxin $(50 \mu \mathrm{M})$ and the tyrosine phosphatase inhibitor sodium orthovanadate $(200 \mu \mathrm{M})$ were included in the bath. Patch pipettes were pulled from 1.5-mm borosilicate glass capillaries (WPI, Sarasota, FL) to a resistance of 3-7 M $\Omega$ with a horizontal puller (Model P97; Sutter Instruments, Novato, CA) and filled with a solution containing (in millimolars): $140 \mathrm{CsCl}, 10 \mathrm{HEPES}, 2 \mathrm{MgCl}_{2}$, $5 \mathrm{Na}^{+}$-ATP, $0.6 \mathrm{Na}^{+}$-ATP, 2 QX314, pH 7.3, 285 mOsm. Evoked postsynaptic currents (eEPSCs) were elicited by local stimulation (20-40 V square pulses, $0.3 \mathrm{~ms}$, delivered every 20 seconds) using a bipolar electrode placed $<300 \mu \mathrm{m}$ lateral to recorded neurons. The minimum amount of stimulation needed to elicit a synaptic response with $<15 \%$ variability in amplitude was used and only neurons with an access resistance of $<30 \mathrm{M} \Omega$ were included. $\alpha$-amino-3-hydroxy-5methyl-4-isoxazolepropionic acid receptor-mediated eEPSCs were recorded at $-70 \mathrm{mV}$ (Kreitzer and Malenka, 2008). NMDARmediated eEPSCs were recorded at $+40 \mathrm{mV}$ in the presence of the $\alpha$-amino-3-hydroxy-5-methyl-4-isoxazolepropionic acid receptor antagonist 6,7-dinitroquinoxaline-2,3-dione $(20 \mu \mathrm{M})$. To confirm that eEPSCs were NMDAR mediated, the NMDAR antagonist (2R)-amino5 -phosphonovaleric acid $(50 \mu \mathrm{M})$ was bath applied at the end of the recording. The contribution of GluN2B-containing NMDARs to the total NMDAR-mediated eEPSC in WT mice was determined using the GluN2B antagonist Ro 25-6981 (0.5 $\mu \mathrm{M}$, 1594; Tocris, Bristol, United Kingdom).
Data Analysis and Statistics. Data and statistical analyses were performed using Excel, GraphPad Prism 6 (GraphPad, San Diego, CA), and Statistica 6.0 software (Statsoft, Tulsa, OK). Electrophysiological data were acquired using pClamp 10.0 (Molecular Devices, Sunnyvale, CA), sampled at $20 \mathrm{kHz}$, filtered at $10 \mathrm{kHz}$, and analyzed in Clampfit 10.4 and Prism 6. All values are reported as mean \pm S.E.M. and the criterion for statistical significance was $P \leq 0.05$. For comparison of three or more factors (between subject's factors: genotype/treatment; within subject's factor: time), repeated measures (RM) analysis of variance (ANOVA) was used, followed by Sidak's post hoc multiple comparisons test when appropriate.

\section{Results}

AC1 Is Required for Ethanol-Induced Locomotor Sensitization. Increases in ambulatory activity (Fig. 1, A and $\mathrm{D}$ ) and the time course of locomotor responding following acute (Fig. 1, B and E) and challenge (Fig. 1, C and F) ethanol exposure were assessed in WT and AC1KO mice (Fig. 1, A-C) and WT mice pretreated with saline or the AC1 inhibitor NB001 (Fig. 1, D-F, $10 \mathrm{mg} / \mathrm{kg}$, i.p.) relative to saline-only controls. Average ambulatory counts on the first saline acclimation day prior to ethanol administration were similar across WT $(132.4 \pm 12.0, n=14)$ and $\mathrm{AC} 1 \mathrm{KO}(126.4 \pm 7.7, n=$ 13) cohorts and saline $(84.7 \pm 8.5, n=18)$ and NB001 (107.4 \pm $6.4, n=17)$ pretreated WT cohorts. Similarly, statistically equivalent reductions in average ambulations across repeated
A

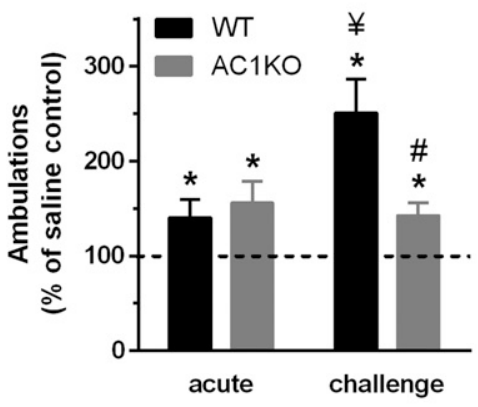

D

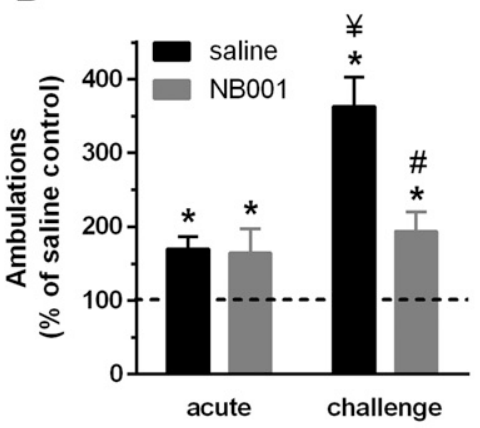

B

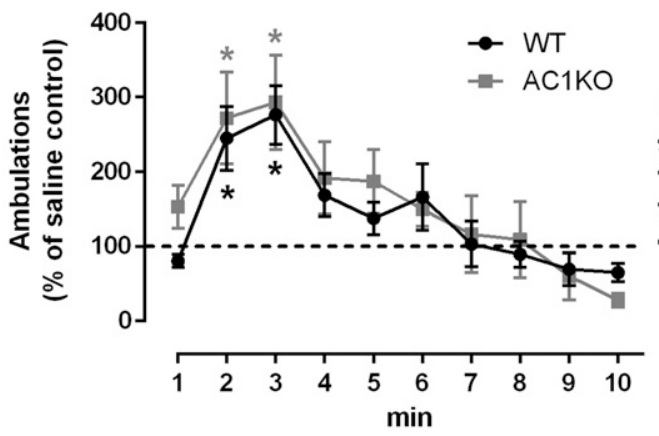

$\mathbf{E}$

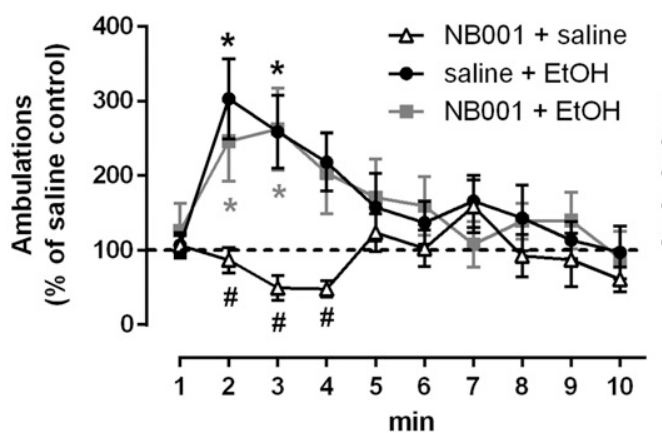

C

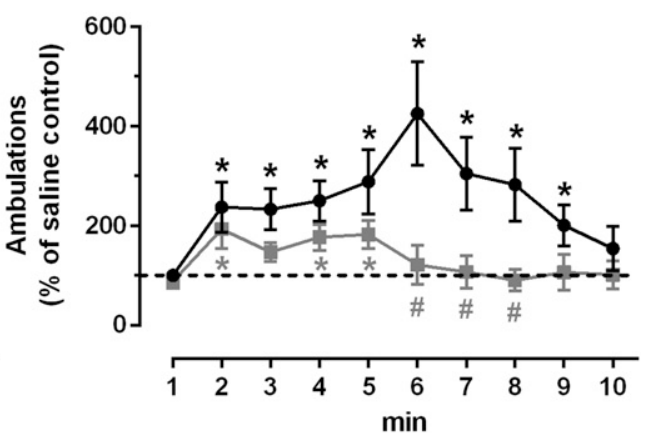

EtOH challenge $(2 \mathrm{~g} / \mathrm{kg})$

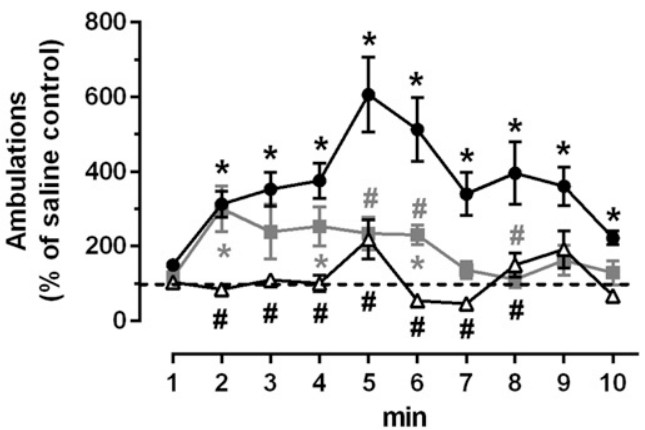

Fig. 1. Intact acute, but absence of sensitized ethanol (EtOH) locomotor response in AC1KO mice (A-C), $n=6-7 /$ group, and WT mice pretreated with the selective inhibitor NB001 (D-F), $10 \mathrm{mg} / \mathrm{kg}, n=8-9 /$ group. Average locomotor activity (A and D) and time course following acute (B and E) or challenge $(\mathrm{C}$ and $\mathrm{F}) \mathrm{EtOH}(2.0 \mathrm{~g} / \mathrm{kg})$ after repeated home cage EtOH administration $(2.5 \mathrm{~g} / \mathrm{kg}, 10$ injections). Data reported as percentage change relative to respective saline-only controls. WT EtOH-treated mice displayed an acute locomotor response relative to saline-only WT controls, which was significantly enhanced following sensitization. AC1KO and NB001-treated WT mice demonstrated a comparable acute locomotor response to WT EtOH controls, but failed to display a further potentiation in this response following sensitization. Locomotor activity on the acute and challenge day was unaltered by pretreatment with NB001 alone (NB001 + saline). Significance determined by two-way RM ANOVA and Sidak's post hoc test, $* P \leq 0.05$, compared with respective saline-treated controls; ${ }^{\sharp} P<0.05$, compared with WT + acute EtOH or saline + acute EtOH group; ${ }^{\#} P<0.05$, compared with $\mathrm{WT}+$ challenge EtOH or saline + acute (E) or challenge (D, F) EtOH group. 
saline acclimation sessions (data not shown) were observed within WT versus AC1KO [two-way RM ANOVA significant effect of time: $\mathrm{F}(2,50)=43.64, P<0.001$; no genotype effect: $\mathrm{F}(1,25)=0.344, P=0.563$; or interaction: $\mathrm{F}(2,50)=2.760, P=$ 0.073] and WT saline versus WT NB001 groups [two-way RM ANOVA significant effect of time: $\mathrm{F}(3,99)=23.99, P<0.001$; no treatment effect: $\mathrm{F}(1,33)=3.418, P=0.0735$; or interaction: $\mathrm{F}(3,99)=1.876, P=0.1386]$. These data indicate the absence of $\mathrm{AC} 1$ does not influence the locomotor response to novelty or induce general motor impairments, consistent with observations following global AC1 gene deficiency (Arakawa et al., 2014) and NB001 treatment (Wang et al., 2011).

Two-way ANOVA comparison of locomotor responses in WT and $\mathrm{AC} 1 \mathrm{KO}$ mice ( $n=6-7 /$ group) following acute and challenge ethanol exposure revealed a significant treatment $\times$ genotype interaction [Fig. 1A; $\mathrm{F}(1,23)=6.509, P=0.017$ ]. WT mice exhibited a transient increase in locomotor activity following acute ethanol $(2.0 \mathrm{~g} / \mathrm{kg}$, i.p.) relative to saline controls (Fig. 1A; WT saline versus WT acute, $P<0.05$ ), which peaked within the first 3 minutes of testing (Fig. 1B). AC1KO mice displayed a comparable acute locomotor response to ethanol (AC1KO saline versus $\mathrm{AC} 1 \mathrm{KO}$ acute, $P<0.05$ ) as observed in WT mice. Repeated ethanol (2.5 g/kg, i.p. 10 daily injections) induced locomotor sensitization in WT mice, as evidenced by an increased ambulatory response to a challenge injection of ethanol at the same dose administered at the acute time point $(2.0 \mathrm{~g} / \mathrm{kg})$ (Fig. 1A; WT acute versus WT challenge, $P<0.05$ ). In contrast, the locomotor stimulation elicited by ethanol challenge in AC1KO mice $\mathrm{AC} 1 \mathrm{KO}$ saline versus AC1KO challenge, $P<$ $0.05)$ was similar to the acute ethanol response in WT $(P=$ $0.999)$ and $\mathrm{AC} 1 \mathrm{KO}$ mice $(P=0.979)$ and was significantly attenuated relative to ethanol-sensitized WT mice (WT challenge versus $\mathrm{AC} 1 \mathrm{KO}$ challenge, $P<0.05$ ).

Pharmacologic inhibition of AC1 activity with NB001 in WT mice resulted in comparable effects on ethanol locomotor responses [Fig. 1D, significant NB001 $\times$ ethanol treatment interaction: $\mathrm{F}(1,30)=7.424, P=0.0106, n=8-9 /$ group] to those observed in AC1KO mice. Mice pretreated with NB001 displayed an acute ethanol locomotor response relative to saline-only controls, which was similar to that observed in saline pretreated WT mice (saline + saline versus saline + acute, $P<$ 0.05; saline + saline versus NB001 + acute, $P<0.05)$. However, the locomotor response to ethanol challenge in NB001 pretreated mice was comparable to that observed with acute ethanol in saline $(P=0.946)$ and NB001 pretreated mice $(P=0.909)$ and was significantly reduced relative to the challenge response in saline pretreated mice (saline + challenge versus NB001+ challenge, $P<0.01$ ). Furthermore, mice with impaired AC1 signaling (AC1KO and NB001 treated) demonstrated a differential pattern of locomotor activity to ethanol challenge [Fig. 1, $\mathrm{C}$ and $\mathrm{F}$; two-way RM ANOVA significant time $\times$ genotype interaction: $\mathrm{F}(9,54)=2.116, P=0.044$; significant time $\times$ treatment interaction: $\mathrm{F}(9,135)=3.620, P=0.0005]$, in that repeated ethanol exposure increased and prolonged peak locomotor responses in WT ethanol controls, but not AC1KO or NB001-treated mice.

Ethanol-Induced Potentiation of GluN2B Phosphorylation in the DMS Is Absent in AC1KO Mice. Since AC1 may promote ethanol-induced behavioral sensitization through recruitment of GluN2B-containing NMDARs, levels of pTyr-1472-GluN2B-NMDAR in DMS lysates were measured following repeated saline or ethanol injections in
AC1KO mice and WT mice pretreated with NB001 (Fig. 2A). Two-way ANOVA analysis of pTyr-1472-GluN2B levels measured 24 hours following the last saline or ethanol injection revealed a significant $\mathrm{AC} 1$ status $\times$ treatment interaction [Fig. 2A; $\mathrm{F}(2,18)=7.522, P=0.004 ; n=3-5$ /group]. An increase in pTyr-1472-GluN2B levels within the DMS was detected in WT mice 24 hours following ethanol-induced sensitization (WT saline versus WT ethanol, $P<0.05$ ). However, this effect was not observed in $\mathrm{AC} 1 \mathrm{KO}$ mice (AC1KO saline versus $\mathrm{AC} 1 \mathrm{KO}$ ethanol, $P=0.883$ ) or WT mice pretreated with NB001 during the entire sensitization procedure (NB001 + saline versus NB001 + ethanol, $P>0.999)$, reflecting their lack of a locomotor sensitization response. Rather, pTyr1472-GluN2B levels in the DMS following repeated ethanol were significantly attenuated in AC1KO and NB001-treated mice compared with that observed in WT mice (both $P<0.05$ ). A similar pattern of changes was observed in the DMS at a more immediate time point (20 minutes) following the sensitization procedure [two-way ANOVA significant AC1 status $\times$ treatment interaction: $\mathrm{F}(1,18)=7.385, P=0.014 ; n=5-6$ group; data not shown]. Here, levels of pTyr-1472-GluN2B were also significantly augmented in WT mice repeatedly treated with ethanol (WT saline versus WT ethanol, $29.0 \% \pm 6.2 \%$ increase, $P>0.05$ ), consistent with prior observations of ethanol-induced increases in pTyr-1472-GluN2B-NMDAR in this region at similar immediate and protracted time points (Wang et al., 2010). Importantly, this effect was absent in ethanol-treated $\mathrm{AC} 1 \mathrm{KO}$ mice (AC1KO saline versus $\mathrm{AC} 1 \mathrm{KO}$ ethanol, 14.3\% \pm $8.3 \%$ decrease, $P=0.361$ ). No changes in total GluN2BNMDARs levels in the DMS were identified at either time point relative to actin.

Since ethanol regulation of GluN2B activity was postulated to be specific for the DMS (Wang et al., 2010), the present study assessed for possible changes in pTyr-1472-GluN2B and total GluN2B levels 24 hours following ethanol sensitization in proximal subregions of the DLS (Fig. 2B) and NAc (Fig. 2C). In the DLS, repeated treatment with ethanol reduced pTyr1472-GluN2B levels similarly across all groups [Fig. 2B; twoway ANOVA significant effect of ethanol treatment: $\mathrm{F}(1,17)=$ 47.68, $P<0.0001$; no effect of AC1 inhibition: $\mathrm{F}(2,17)=3.369$, $P=0.059$; or interaction: $\mathrm{F}(2,17)=0.474, P=0.630]$. Post hoc analysis confirmed that levels of pTyr-1472-GluN2B were significantly attenuated in ethanol-treated WT, AC1KO, and NB001-treated mice, relative to saline-treated controls (all $P<0.05$ ). Conversely, no changes in p Tyr-1472-GluN2B levels were detected in the NAc [Fig. 2C; two-way ANOVA no effect of ethanol treatment: $\mathrm{F}(1,18)=0.008, P=0.928$; AC1 inhibition: $\mathrm{F}(2,18)=1.608, P=0.228$; or interaction: $\mathrm{F}(2,18)=$ $0.101, P=0.904]$. Also, no significant changes in total GluN2B levels normalized to actin were identified in the DLS or NAc, irrespective of ethanol treatment or AC1 activity status.

Ethanol Increases NMDAR-Mediated Transmission in the DMS of WT, but Not AC1KO, Mice. Phosphorylation of Tyr-1472 is known to increase the synaptic localization and transmission of GluN2B-NMDARs (Prybylowski et al., 2005; Wang et al., 2010). To determine whether loss of AC1 also prevented ethanol-induced increases in NMDAR-mediated transmission, whole-cell patch-clamp recordings in the DMS were performed 20-26 hours following the last ethanol treatment (Fig. 3, A-D). Stimulation intensities needed to elicit a response did not differ between groups and access resistance was similar between groups. Consistent with previous reports 


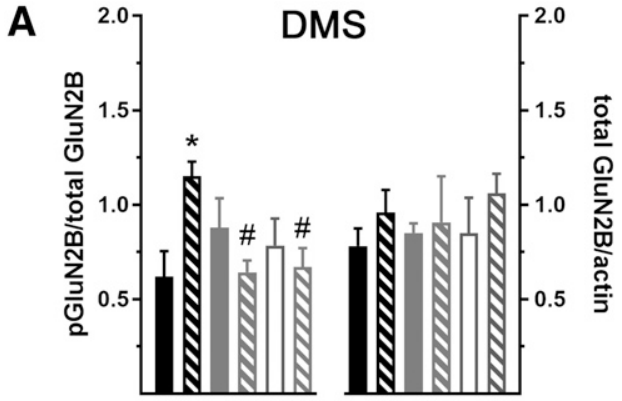

B

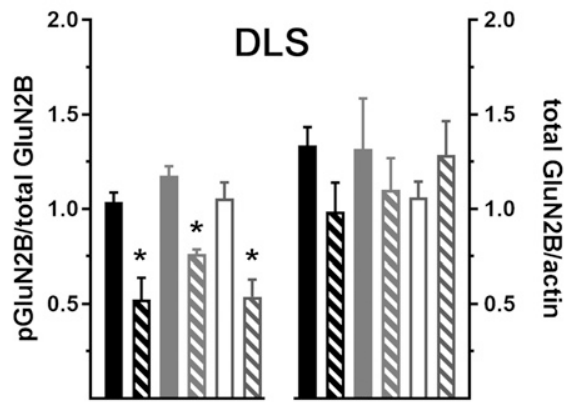

C

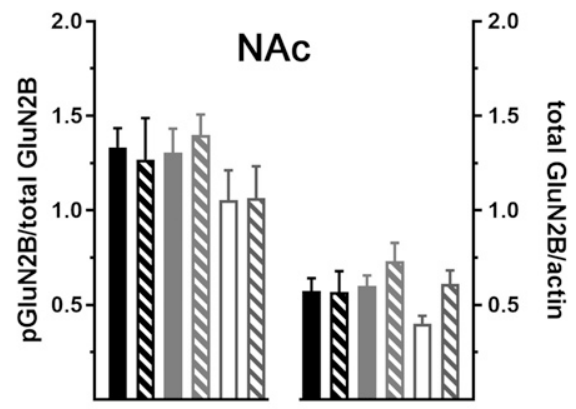

WT sal $\quad$ AC1KO sal $\square$ NB001 sal

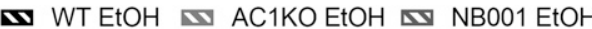
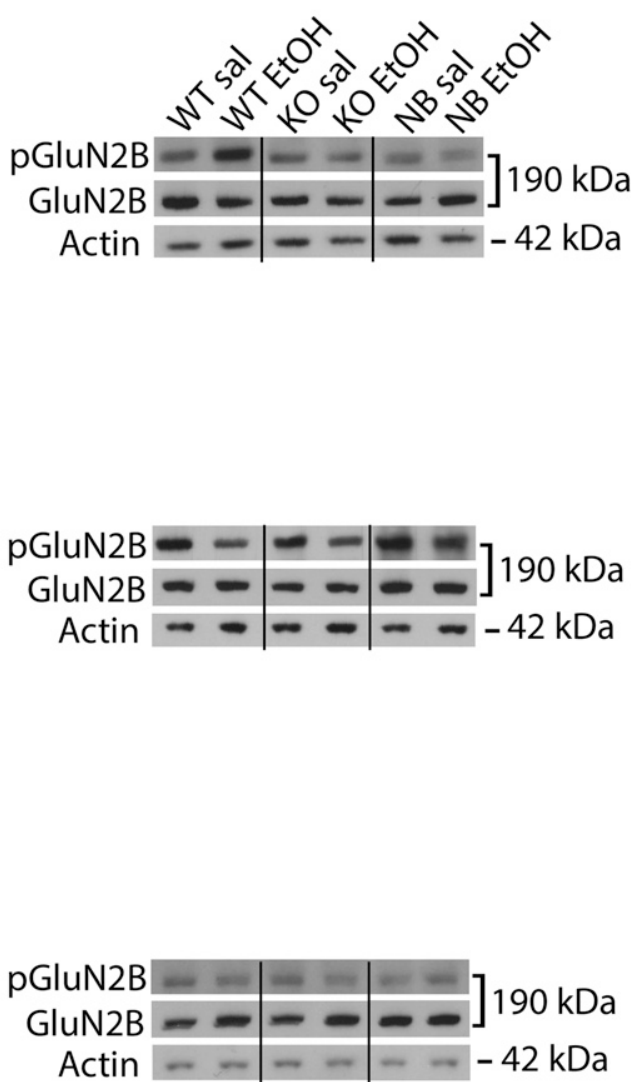

Fig. 2. Repeated ethanol (EtOH) selectively enhanced GluN2B-NMDAR phosphorylation in the DMS in WT, but not AC1KO (KO) mice or mice pretreated with NB001 ( $n=3-5$ /group). Averaged data (left) and representative immunoblots compiled from groups of images from different parts of the same gel (right) are depicted for DMS (A), DLS (B), or NAc (C) lysates prepared following repeated saline (sal) or $\mathrm{EtOH}$ treatment and probed for total and pTyr-1472-GluN2B. (A) A significant increase in pGluN2B levels in the DMS of WT mice was observed following repeated $\mathrm{EtOH}$ treatment relative to saline-treated controls, which was absent in AC1KO and NB001 pretreated mice. (B) Repeated ethanol treatment decreased levels of pGluN2B in the DLS equivalently across WT, AC1KO, and NB001-treated mice. (C) No changes in pGluN2B levels in the NAc were observed in response to ethanol or absence of AC1 signaling. Total GluN2B levels were unaffected by AC1 status or ethanol treatment in all three regions. Significance determined by two-way ANOVA and Sidak's post hoc test, $* P<$ 0.05 , compared with saline-treated controls; ${ }^{\#} P<0.05$, compared with WT EtOH.
(Wang et al., 2010), ethanol increased NMDAR-mediated transmission in WT mice [Fig. 3, A and B; two-way ANOVA significant group $\times$ treatment interaction: $\mathrm{F}(1,40)=4.842, P=$ 0.034; WT saline versus WT ethanol, $P<0.05$; WT saline $N=$ 15 cells from nine mice; WT ethanol $N=13$ cells from seven mice]. In contrast, NMDAR-mediated transmission did not differ between saline- and ethanol-treated AC1KO mice (Fig. 3, $\mathrm{A}$ and $\mathrm{B}$; AC1KO saline versus AC1KO ethanol, $P>0.05$; AC1KO saline $N=8$ cells from six mice; AC1KO ethanol $N=$ 8 cells from five mice). In addition, NMDAR-mediated transmission was similar between saline-treated WT and AC1KO mice (Fig. 3B).

The contribution of GluN2B-containing receptors was determined in WT mice treated with saline and ethanol by bath application of Ro 25-6981 (0.5 $\mu \mathrm{M})$. In saline-treated WT mice, $\sim 20 \%$ inhibition of GluN2B-containing NMDAR-mediated transmission was observed with Ro 25-6981 compared with $\sim 40 \%$ reduction in ethanol-treated mice (Fig. $3 \mathrm{C}$ ), similar to that previously reported (Wang et al., 2010). ANOVA analysis confirmed a potentiated inhibition of NMDAR-mediated transmission with Ro 25-6981 in ethanol-treated mice [twoway RM ANOVA significant group $\times$ treatment interaction: $\mathrm{F}(1,11)=11.33, P=0.0063$; WT ethanol: baseline versus + Ro 25-6981, $P<0.05$; WT saline: baseline versus + Ro 25-6981, $P>0.05$; WT ethanol: baseline $N=7$ cells from four mice; WT saline: baseline $N=6$ cells from four mice). Furthermore, addition of Ro 25-6981 reduced NMDAR-mediated transmission in ethanol-treated WT mice to levels comparable to saline controls, supporting the idea that the ethanol-induced increase in NMDAR transmission was almost entirely due to GluN2B-containing NMDARs. Together, these data show that ethanol enhances NMDAR-mediated transmission by increasing the contribution of GluN2B-containing receptors. These data are consistent with the immunoblot results and a previous report (Wang et al., 2010). In addition, our biochemical and electrophysiological data demonstrate that AC1KO prevents ethanol-induced increases in NMDARmediated transmission and phosphorylation, corresponding with impaired locomotor sensitization responding.

\section{Discussion}

The present study reveals a pivotal role for the calcium/ calmodulin-stimulated cyclase enzyme AC1 in the sensitized behavioral effects and GluN2B-NMDAR neuroadaptations in the DMS induced by repeated ethanol exposure. Loss of AC1 signaling, by genetic deletion or pharmacological inhibition, resulted in a specific impairment of ethanol-induced locomotor sensitization, while baseline and acute ethanol locomotor responses were unaffected. Repeated exposure to ethanol in 
A Example NMDAR-mediated eEPSCs
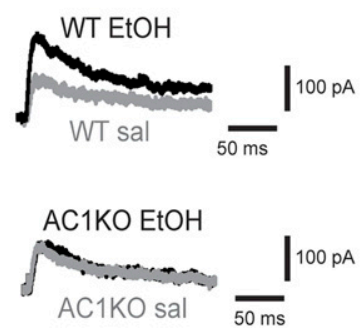

C EtOH increases the contribution of GluN2B-containing NMDARs

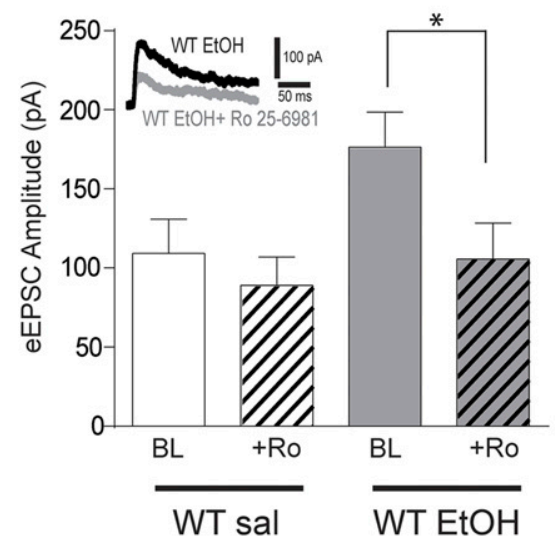

\section{B NMDAR-mediated current}

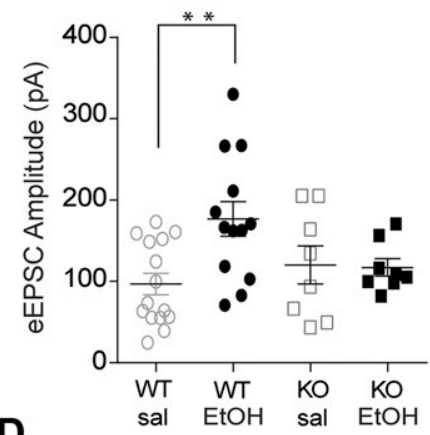

D

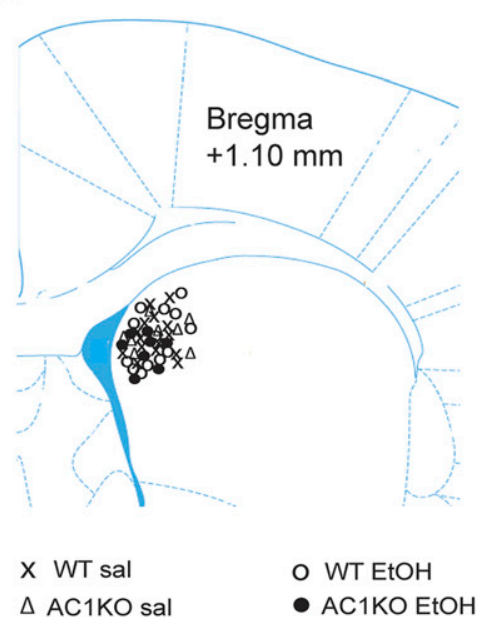

Fig. 3. Ethanol (EtOH) upregulation of NMDAR-mediated transmission is absent in $\mathrm{AC} 1 \mathrm{KO}$ (KO) mice. (A) Representative traces of NMDAR-mediated eEPSCs and (B) average NMDAR-mediated eEPSC amplitude ( \pm S.E.M.) in WT and AC1KO mice repeatedly treated with saline (sal) or EtOH. EtOH produced an increase in NMDAR-mediated eEPSC amplitude only in WT mice (two-way ANOVA and Sidak's post hoc test, ${ }^{* *} P<0.01$ ). (C) Average NMDAR-mediated eEPSC amplitude before (BL) and after (+Ro) bath application of the GluN2B selective antagonist Ro 25-6981 $(0.5 \mu \mathrm{M})$ in WT mice. EtOH-induced upregulation of NMDAR-mediated transmission involved an increased contribution of GluN2Bcontaining NMDARs (two-way RM ANOVA and Sidak's post hoc test, ${ }^{*} P<0.05$ ). (D) Average central location of medium spiny neuron recordings was $1.10 \mathrm{~mm}$ from bregma $( \pm 0.5 \mathrm{~mm})$. the sensitization procedure enhanced striatal GluN2BNMDAR tyrosine phosphorylation and promoted upregulation in NMDAR transmission in WT mice in the DMS. Loss of AC1 signaling impaired both ethanol-induced GluN2BNMDAR modifications in the DMS, but did not alter the reduction in pTyr-1472-GluN2B observed in the DLS with ethanol treatment. Thus, our data establish AC1 as a critical facilitator of NMDAR signaling that may underlie neuroplastic responses induced in DMS pathways by repeated ethanol exposure.

Striatal NMDARs are critical for the neural plasticity underlying ethanol-induced responses, such as behavioral sensitization (Kotlinska et al., 2006; Abrahao et al., 2013). In the present study, the sensitized locomotor response to repeated ethanol in WT mice was accompanied by immediate (20 minutes) and protracted (24 hours) increases in tyrosine phosphorylation of GluN2B-containing NMDARs specifically in the DMS. This lasting biochemical modification corresponded with an upregulation in DMS GluN2B-NMDAR activity measured 20-26 hours following the last administration of ethanol. Our findings are consistent with the previously reported increase in NMDAR phosphorylation and transmission following acute (30 minutes) and extended (16-40 hours) withdrawal from ethanol in this region (Wang et al., 2010).

Furthermore, our present observations reveal that the potentiation of GluN2B phosphorylation induced during protracted stages of early withdrawal from in vivo, repeated ethanol was only evident in the DMS, but not the DLS or NAc, in which either a significant decrease or no change in GluN2B phosphorylation was observed, respectively. The stimulant and sensitizing properties of drugs of abuse are largely thought to rely on signaling in the NAc; however, behavioral sensitization is also associated with enhanced stimulus-response (habit) learning (Nelson and Killcross, 2006; Nordquist et al., 2007), a function primarily attributed to dorsal regions of the striatum (Belin et al., 2009; Volkow et al., 2011). Our findings support and extend previous evidence demonstrating the importance of dorsal striatal glutamatergic transmission in drug-induced behavioral sensitization (Parikh et al., 2014), by indicating a specific role for NMDAR neuroadaptations in the DMS in this process. Interestingly, studies in the NAc indicate measures of NMDAR expression and function were significantly reduced in ethanol-sensitized mice after a prolonged withdrawal period (11-20 days) (Abrahao et al., 2013), which in light of our data indicates regional and temporal differences among sensitizationrelated alterations in NMDAR function.

The biologic significance of the novel finding for an inverse effect of ethanol on GluN2B phosphorylation in the DLS requires further investigation; however, this response was equivalent across all cohorts, supporting the region specificity of $\mathrm{AC} 1$ in ethanol regulation of NMDAR signaling in the DMS. Since recruitment of the DLS has been implicated in habit formation, our findings are in line with the suggestion that passive drug administration does not activate habit systems to the same extent as self-administration (Jacobs et al., 2003; Fanelli et al., 2013). Overall, the correspondence among ethanol-induced NMDAR adaptations in the DMS shown to contribute to compulsive ethanol-seeking behavior (Wang et al., 2010) and those presently observed to accompany sensitization support a fundamental role for glutamatergic 
circuits in the overlapping neurochemical mechanisms underlying behavioral sensitization and drug dependence (Hunt and Lands, 1992; Steketee and Kalivas, 2011), thus representing a mechanistic correlate associated with alcoholism vulnerability.

The neuronal response to ethanol is mediated, in part, through calcium-dependent PKA activation (Ron and Messing, 2013; Baliño et al., 2014). The observations that calciumstimulated ACs are selectively elevated in striatal limbic areas of brains from human alcoholics (Yamamoto et al., 2001) and are imperative for ethanol-mediated PKA phosphorylation of synaptic targets (Conti et al., 2009) are consistent with a scenario in which ethanol recruitment of these enzymes mediates the maladaptive striatal signaling response to ethanol. While other $\mathrm{AC}$ isoforms were shown to participate in the neurobehavioral response to ethanol, including AC7 (Cruz et al., 2011), only AC1 and AC8 have the distinct regulatory characteristics to modulate ethanolinduced PKA signaling that is dependent on intracellular calcium (Baliño et al., 2014). Our finding that mice lacking AC1 failed to develop a sensitized locomotor response to ethanol supports our hypothesis that $\mathrm{AC} 1$ is recruited as part of the neuroadaptations associated with drug dependence, consistent with its upregulation in striatal tissue from human alcoholics (Yamamoto et al., 2001). The involvement of AC1 in ethanol-induced locomotor sensitization was mechanistically validated in the present study by pharmacological inhibition with NB001, which blocks AC1-stimulated cAMP production in mouse brain slices and human neurons with greater than 10-fold AC isoform selectivity relative to AC5-8 (Wang et al., 2011). In support of its selectivity, Wang et al. (2011) reported that the dose of NB001 used in the present study $(10 \mathrm{mg} / \mathrm{kg})$ failed to influence anxiety or general motor behavior, which has been largely attributed to the other AC isoforms (AC5-8) (Iwamoto et al., 2003; Kim et al., 2008; Razzoli et al., 2010; Cruz et al., 2011). While a subsequent study suggested NB001 does not directly inhibit AC1 activity (Brand et al., 2013), the authors reported that NB001-mediated inhibition of cAMP accumulation still occurred in an AC1-dependent manner; thereby, not precluding its utility to dissect pathways specifically activated by AC1. Furthermore, the absence of basal and acute ethanol locomotor effects in AC1KO and NB001-treated mice indicates the loss of AC1 does not impair ethanol sensitization responding through a general suppression of behavior or increased sensitivity to the sedative effects of ethanol. Greater ambulatory responses to stimulants than presently observed with acute ethanol have been reported in mice lacking calcium-stimulated ACs (DiRocco et al., 2009; Bosse et al., 2015), further indicating that the absence of an ethanol sensitization response in AC1KO mice is not likely due to a ceiling effect on locomotor stimulation.

Our finding of impaired ethanol-induced sensitization in $\mathrm{AC} 1 \mathrm{KO}$ mice in the absence of acute locomotor changes suggests AC1 predominately facilitates the neuroplastic processes evoked by repeated ethanol. In line with this, genetic deletion and pharmacologic inhibition of AC1 prevented the ethanol-induced increases in GluN2B phosphorylation observed during protracted stages of early withdrawal (24 hours) within the DMS in WT mice. This effect coincided with reduced DMS NMDAR-mediated transmission in the absence of AC1 at a similar time point following ethanol administration. Phosphorylation of GluN2B at Tyr-1472 increases NMDAR synaptic expression (Prybylowski et al., 2005), which likely contributes to the long-lasting potentiation of GluN2B-NMDAR transmission in response to repeated ethanol exposure. Striatal pTyr-1472-GluN2B levels are dynamically regulated by Fyn kinase and striatal-enriched protein tyrosine phosphatase 61. Enhanced PKA-mediated inhibition of striatal-enriched protein tyrosine phosphatase 61 activity following repeated ethanol exposure was shown to be sufficient for ethanol activation of Fyn kinase and GluN2B phosphorylation (Darcq et al., 2014). Since calcium was shown to be a cellular mediator of ethanol activation of PKA (Baliño et al., 2014), we propose that ethanol initiation of PKA signaling in this pathway represents a mechanistic target for $\mathrm{AC} 1$ regulation in the neuroadaptive response to repeated ethanol.

In conclusion, our findings identify $\mathrm{AC} 1$ as a key modulator of sensitization-related alterations in DMS GluN2B-NMDARs induced by repeated ethanol exposure. Drug sensitization and associated striatal neuroadaptations have also been observed in humans (Boileau et al., 2003; O'Daly et al., 2014; Grodin et al., 2016), which support the validity of this preclinical model to recapitulate signaling adaptations that promote drug responding. Since AC1 activity is stimulated by synapticspecific increases in calcium, our evidence would support its unique role in translating activity-dependent signals into aberrant neuronal and behavioral responses to ethanol. While NMDAR-based pharmacotherapies for alcoholism have shown promise clinically, side effects associated with the ubiquitous expression and physiologic importance of NMDARs limit their therapeutic potential (Holmes et al., 2013; Spanagel and Vengeliene, 2013). Targets that modify these processes under conditions conducive for plasticity (e.g., activity-dependent calcium increase) may offer sufficient specificity for therapy development. Future pharmacologic studies targeting AC1 in models of ethanol consumption would further support its translational relevance in rehabilitative strategies designed for the affected population.

\section{Acknowledgments}

The authors thank Dr. Shane Perrine and Dr. Matthew Galloway for the use of the locomotor activity monitors and Arman Harutyunyan for assistance with the NB001 sensitization experiment.

\section{Authorship Contributions}

Participated in research design: Bosse, Oginsky, Ferrario, Conti. Conducted experiments: Bosse, Oginsky, Susick, Ramalingham, Ferrario, Conti.

Performed data analysis: Bosse, Oginsky, Susick, Ramalingham, Ferrario, Conti.

Wrote or contributed to the writing of the manuscript: Bosse, Oginsky, Ferrario, Conti.

\section{References}

Abrahao KP, Ariwodola OJ, Butler TR, Rau AR, Skelly MJ, Carter E, Alexander NP, McCool BA, Souza-Formigoni ML, and Weiner JL (2013) Locomotor sensitization to ethanol impairs NMDA receptor-dependent synaptic plasticity in the nucleus accumbens and increases ethanol self-administration. $J$ Neurosci 33:4834-4842. Arakawa H, Akkentli F, and Erzurumlu RS (2014) Region-specific disruption of adenylate cyclase type 1 gene differentially affects somatosensorimotor behaviors in mice. eNeuro 1 DOI: http://dx.doi.org/10.1523/ENEURO.0007-14.2014.

Baliño P, Ledesma JC, and Aragon CM (2014) In vivo study of ethanol-activated brain protein kinase A: manipulations of $\mathrm{Ca}^{2+}$ distribution and flux. Alcohol Clin Exp Res 38:629-640.

Belin D, Jonkman S, Dickinson A, Robbins TW, and Everitt BJ (2009) Parallel and interactive learning processes within the basal ganglia: relevance for the understanding of addiction. Behav Brain Res 199:89-102. 
Boileau I, Assaad JM, Pihl RO, Benkelfat C, Leyton M, Diksic M, Tremblay RE, and Dagher A (2003) Alcohol promotes dopamine release in the human nucleus accumbens. Synapse 49:226-231.

Bosse KE, Charlton JL, Susick LL, Newman B, Eagle AL, Mathews TA, Perrine SA, and Conti AC (2015) Deficits in behavioral sensitization and dopaminergic responses to methamphetamine in adenylyl cyclase 1/8-deficient mice. $J$ Neurochem 135:1218-1231.

Brand CS, Hocker HJ, Gorfe AA, Cavasotto CN, and Dessauer CW (2013) Isoform selectivity of adenylyl cyclase inhibitors: characterization of known and novel compounds. J Pharmacol Exp Ther 347:265-275.

Cabib S, Puglisi-Allegra S, and Ventura R (2002) The contribution of comparative studies in inbred strains of mice to the understanding of the hyperactive phenotype. Behav Brain Res 130:103-109.

Conti AC, Lowing JL, Susick LL, and Bowen SE (2012) Investigation of calciumstimulated adenylyl cyclases 1 and 8 on toluene and ethanol neurobehavioral actions. Neurotoxicol Teratol 34:481-488.

Conti AC, Maas JW, Jr, Moulder KL, Jiang X, Dave BA, Mennerick S, and Muglia LJ (2009) Adenylyl cyclases 1 and 8 initiate a presynaptic homeostatic response to ethanol treatment. PLoS One 4:e5697.

Crabbe JC, Jr, Johnson NA, Gray DK, Kosobud A, and Young ER (1982) Biphasic effects of ethanol on open-field activity: sensitivity and tolerance in C57BL/6N and DBA/2N mice. J Comp Physiol Psychol 96:440-451.

Cruz MT, Bajo M, Maragnoli ME, Tabakoff B, Siggins GR, and Roberto M (2011) Type 7 adenylyl cyclase is involved in the ethanol and CRF sensitivity of GABAergic synapses in mouse central amygdala. Front Neurosci 4:207.

Darcq E, Hamida SB, Wu S, Phamluong K, Kharazia V, Xu J, Lombroso P, and Ron D (2014) Inhibition of striatal-enriched tyrosine phosphatase 61 in the dorsomedial striatum is sufficient to increased ethanol consumption. $J$ Neurochem 129 1024-1034.

DiRocco DP, Scheiner ZS, Sindreu CB, Chan GC, and Storm DR (2009) A role for calmodulin-stimulated adenylyl cyclases in cocaine sensitization. $J$ Neurosci 29 2393-2403.

Fanelli RR, Klein JT, Reese RM, and Robinson DL (2013) Dorsomedial and dorsolateral striatum exhibit distinct phasic neuronal activity during alcohol selfadministration in rats. Eur $J$ Neurosci 38:2637-2648.

Grodin EN, Steckler LE, and Momenan R (2016) Altered striatal response during effort-based valuation and motivation in alcohol-dependent individuals. Alcohol Alcohol 51:638-646.

Hefner K and Holmes A (2007) An investigation of the behavioral actions of ethanol across adolescence in mice. Psychopharmacology (Berl) 191:311-322.

Holmes A, Spanagel R, and Krystal JH (2013) Glutamatergic targets for new alcohol medications. Psychopharmacology (Berl) 229:539-554.

Hunt WA and Lands WE (1992) A role for behavioral sensitization in uncontrolled ethanol intake. Alcohol 9:327-328.

Iwamoto T, Okumura S, Iwatsubo K, Kawabe J, Ohtsu K, Sakai I, Hashimoto Y, Izumitani A, Sango K, Ajiki K, et al. (2003) Motor dysfunction in type 5 adenylyl cyclase-null mice. J Biol Chem 278:16936-16940.

Jacobs EH, Smit AB, de Vries TJ, and Schoffelmeer AN (2003) Neuroadaptive effects of active versus passive drug administration in addiction research. Trends Pharmacol Sci 24:566-573.

Kim KS, Lee KW, Baek IS, Lim CM, Krishnan V, Lee JK, Nestler EJ, and Han PL (2008) Adenylyl cyclase-5 activity in the nucleus accumbens regulates anxietyrelated behavior. J Neurochem 107:105-115.

Kotlinska J, Bochenski M, and Danysz W (2006) N-methyl-D-aspartate and group I metabotropic glutamate receptors are involved in the expression of ethanolinduced sensitization in mice. Behav Pharmacol 17:1-8.

Kreitzer AC and Malenka RC (2008) Striatal plasticity and basal ganglia circuit function. Neuron 60:543-554.

Lessov CN, Palmer AA, Quick EA, and Phillips TJ (2001) Voluntary ethanol drinking in C57BL/6.J and DBA/2.J mice before and after sensitization to the locomotor stimulant effects of ethanol. Psychopharmacology (Berl) 155 91-99.
Maas JW, Jr, Vogt SK, Chan GC, Pineda VV, Storm DR, and Muglia LJ (2005) Calcium-stimulated adenylyl cyclases are critical modulators of neuronal ethanol sensitivity. J Neurosci 25:4118-4126.

Melón LC and Boehm SL, II (2011) Role of genotype in the development of locomotor sensitization to alcohol in adult and adolescent mice: comparison of the DBA/2J and C57BL/6J inbred mouse strains. Alcohol Clin Exp Res 35:1351-1360.

Nelson A and Killcross S (2006) Amphetamine exposure enhances habit formation. $J$ Neurosci 26:3805-3812.

Nordquist RE, Voorn P, de Mooij-van Malsen JG, Joosten RN, Pennartz CM, and Vanderschuren LJ (2007) Augmented reinforcer value and accelerated habit formation after repeated amphetamine treatment. Eur Neuropsychopharmacol 17:532-540. O’Daly OG, Joyce D, Tracy DK, Azim A, Stephan KE, Murray RM, and Shergill SS (2014) Amphetamine sensitization alters reward processing in the human striatum and amygdala. PLoS One 9:e93955.

Olsen CM, Huang Y, Goodwin S, Ciobanu DC, Lu L, Sutter TR, and Winder DG (2008) Microarray analysis reveals distinctive signaling between the bed nucleus of the stria terminalis, nucleus accumbens, and dorsal striatum. Physiol Genomics 32:283-298.

Parikh V, Naughton SX, Shi X, Kelley LK, Yegla B, Tallarida CS, Rawls SM, and Unterwald EM (2014) Cocaine-induced neuroadaptations in the dorsal striatum: glutamate dynamics and behavioral sensitization. Neurochem Int 75:54-65.

Paxinos G and Franklin KBJ (2001) The Mouse Brain, in Stereotaxic Coordinates, Academic Press, San Diego, CA.

Prybylowski K, Chang K, Sans N, Kan L, Vicini S, and Wenthold RJ (2005) The synaptic localization of NR2B-containing NMDA receptors is controlled by interactions with PDZ proteins and AP-2. Neuron 47:845-857.

Razzoli M, Andreoli M, Maraia G, Di Francesco C, and Arban R (2010) Functional role of calcium-stimulated adenylyl cyclase 8 in adaptations to psychological stressors in the mouse: implications for mood disorders. Neuroscience 170:429-440.

Ron D and Messing RO (2013) Signaling pathways mediating alcohol effects. Curr Top Behav Neurosci 13:87-126.

Rose JH, Calipari ES, Mathews TA, and Jones SR (2013) Greater ethanol-induced locomotor activation in $\mathrm{DBA} / 2 \mathrm{~J}$ versus $\mathrm{C} 57 \mathrm{BL} / 6 \mathrm{~J}$ mice is not predicted by presynaptic striatal dopamine dynamics. PLoS One 8:e83852.

Spanagel R and Vengeliene V (2013) New pharmacological treatment strategies for relapse prevention. Curr Top Behav Neurosci 13:583-609.

Steketee JD and Kalivas PW (2011) Drug wanting: behavioral sensitization and relapse to drug-seeking behavior. Pharmacol Rev 63:348-365.

Volkow ND, Wang GJ, Fowler JS, Tomasi D, and Telang F (2011) Addiction: beyond dopamine reward circuitry. Proc Natl Acad Sci USA 108:15037-15042.

Wang H and Storm DR (2003) Calmodulin-regulated adenylyl cyclases: cross-talk and plasticity in the central nervous system. Mol Pharmacol 63:463-468.

Wang H, Xu H, Wu LJ, Kim SS, Chen T, Koga K, Descalzi G, Gong B, Vadakkan KI, Zhang X, et al. (2011) Identification of an adenylyl cyclase inhibitor for treating neuropathic and inflammatory pain. Sci Transl Med 3:65ra3.

Wang J, Lanfranco MF, Gibb SL, Yowell QV, Carnicella S, and Ron D (2010) Longlasting adaptations of the NR2B-containing NMDA receptors in the dorsomedial striatum play a crucial role in alcohol consumption and relapse. $J$ Neurosci $\mathbf{3 0}$ 10187-10198.

Yamamoto M, Pohli S, Durany N, Ozawa H, Saito T, Boissl KW, Zöchling R, Riederer P, Böning J, and Götz ME (2001) Increased levels of calcium-sensitive adenylyl cyclase subtypes in the limbic system of alcoholics: evidence for a specific role of cAMP signaling in the human addictive brain. Brain Res 895:233-237.

Yoshimura M and Tabakoff B (1995) Selective effects of ethanol on the generation of cAMP by particular members of the adenylyl cyclase family. Alcohol Clin Exp Res 19:1435-1440.

Address correspondence to: Alana C. Conti, Department of Neurosurgery, Wayne State University, 4646 John R Street, Detroit, MI 48201. E-mail: alana. conti@wayne.edu 